\title{
On the meromorphic solutions of some linear difference equations
}

\author{
Huifang Liu ${ }^{1 *}$ and Zhiqiang Mao ${ }^{2^{*}}$
}

\author{
"Correspondence: \\ liuhuifang73@sina.com; \\ maozhiqiang1@sina.com \\ 1 Institute of Mathematics and \\ Informatics, Jiangxi Normal \\ University, Nanchang, 330022, China \\ ${ }^{2}$ School of Mathematics and \\ Computer, Jiangxi Science and \\ Technology Normal University, \\ Nanchang, 330038, China
}

\begin{abstract}
This paper is devoted to studying the growth of meromorphic solutions of some linear difference equations. We obtain some results on the growth of meromorphic solutions when most coefficients in such equations have the same order, which are supplements of previous results due to Chiang and Feng, and Laine and Yang. Some examples are given to show the sharpness of our results.
\end{abstract}

MSC: 39B32; 30D35; 39A10

Keywords: complex difference equation; meromorphic solution; growth

\section{Introduction and main results}

In this paper, the term meromorphic function will mean meromorphic in the whole complex plane $\mathbb{C}$. It is assumed that the reader is familiar with the standard notations and basic results of Nevanlinna theory (see, e.g., [1-3]). In addition, we use $\sigma(f)$ and $\sigma_{2}(f)$ to denote the order and the hyper-order of a meromorphic function $f(z)$, and $\lambda(f)$ and $\lambda(1 / f)$ to denote the exponents of convergence of zeros and poles of $f(z)$, respectively. For a meromorphic function $f(z)$, when $0<\sigma(f)<\infty$ or $0<\sigma_{2}(f)<\infty$, its type $\tau(f)$ and hyper-type $\tau_{2}(f)$ are defined by $\tau(f)=\varlimsup_{r \rightarrow \infty} \frac{T(r, f)}{r^{\sigma(f)}}$ and $\tau_{2}(f)=\varlimsup_{r \rightarrow \infty} \frac{\log T(r, f)}{r^{\sigma_{2}(f)}}$ (see, e.g., [1, 2, 4]).

Recently, meromorphic solutions of complex difference equations have become a subject of great interest from the viewpoint of Nevanlinna theory, due to the apparent role of the existence of such solutions of finite order for the integrability of discrete difference equations (see, e.g., [5-19]). Halburd and Korhonen [13] proved that when the difference equation

$$
\omega(z+1)+\omega(z-1)=R(z, \omega)
$$

where $R(z, \omega)$ is rational in both of its arguments and has an admissible meromorphic solution of finite order, then either $\omega$ satisfies a difference Riccati equation, or equation (1.1) can be transformed by a linear change in $\omega(z)$ to a difference Painlevé equation or a linear difference equation. Thus the linear difference equation plays an important role in the study of properties of difference equations.

Chiang and Feng [8] considered the linear difference equation

$$
a_{k}(z) f(z+k)+\cdots+a_{1}(z) f(z+1)+a_{0}(z) f(z)=0
$$

and obtained the following results.

(c) 2013 Liu and Mao; licensee Springer. This is an Open Access article distributed under the terms of the Creative Commons Attribution License (http://creativecommons.org/licenses/by/2.0), which permits unrestricted use, distribution, and reproduction in any medium, provided the original work is properly cited. 
Theorem A [8] Let $a_{0}(z), \ldots, a_{k}(z)$ be polynomials. If there exists an integer $l(0 \leq l \leq k)$ such that

$$
\operatorname{deg}\left(a_{l}\right)>\max _{\substack{0 \leq j \leq k \\ j \neq l}}\left\{\operatorname{deg}\left(a_{j}\right)\right\}
$$

holds, then every meromorphic solution $f(\not \equiv 0)$ of Eq. (1.2) satisfies $\sigma(f) \geq 1$, where $\operatorname{deg}\left(a_{l}\right)$ denotes the degree of the polynomial $a_{l}$.

Theorem B [8] Let $a_{0}(z), \ldots, a_{k}(z)$ be entire functions. If there exists an integer $l(0 \leq l \leq n)$ such that

$$
\sigma\left(a_{l}\right)>\max _{\substack{0 \leq j \leq k \\ j \neq l}}\left\{\sigma\left(a_{j}\right)\right\}
$$

holds, then every meromorphic solution $f(\not \equiv 0)$ of Eq. (1.2) satisfies $\sigma(f) \geq \sigma\left(a_{l}\right)+1$.

Note that in Theorems A and B, Eq. (1.2) has only one dominating coefficient $a_{l}$. For the case when there is no dominating coefficient and all coefficients are polynomials in Eq. (1.2), Chen [10] obtained an improvement of Theorem A.

Theorem C $[10]$ Let $a_{0}(z), \ldots, a_{k}(z)$ be polynomials such that

$$
\operatorname{deg}\left(a_{0}+\cdots+a_{k}\right)=\max _{0 \leq j \leq k}\left\{\operatorname{deg} a_{j}\right\} \geq 1
$$

Then every finite order meromorphic solution $f(\not \equiv 0)$ of Eq. (1.2) satisfies $\sigma(f) \geq 1$.

For the case when there is more than one of coefficients which have the maximal order, Laine and Yang [18] obtained the following result.

Theorem D [18] Let $a_{0}(z), \ldots, a_{k}(z)$ be entire functions of finite order such that among those having the maximal order $\sigma=\max _{0 \leq j \leq k}\left\{\sigma\left(a_{j}\right)\right\}$, exactly one has its type strictly greater than the others. Then for every meromorphic solution $f(\equiv 0)$ of Eq. (1.2), we have $\sigma(f) \geq \sigma+1$.

Note that in Theorem D, the condition that exactly one coefficient has the maximal type among those coefficients having the maximal order, guarantees that every meromorphic solution $f(\not \equiv 0)$ of Eq. (1.2) satisfies $\sigma(f) \geq \max _{0 \leq j \leq k}\left\{\sigma\left(a_{j}\right)\right\}+1$. The following example shows that when there exists more than one coefficient having the maximal type among those coefficients having the maximal order, $\sigma(f) \geq \max _{0 \leq j \leq k}\left\{\sigma\left(a_{j}\right)\right\}+1$ may hold.

Example 1.1 The difference equation

$$
e^{z+1} f(z+1)-e^{-z} f(z)=0
$$

admits an entire solution $f(z)=e^{-z^{2}}$, where $a_{1}(z)=e^{z+1}, a_{0}(z)=-e^{-z}$ satisfy $\sigma\left(a_{1}\right)=\sigma\left(a_{0}\right)=$ $1=\sigma, \tau\left(a_{1}\right)=\tau\left(a_{0}\right)$. Here $\sigma(f)=2=\sigma+1$.

Thus we pose the following questions. 
Question 1.1 What can be said if there exists more than one coefficient having the maximal type and the maximal order in Eq. (1.2)?

Question 1.2 What can be said if all coefficients of Eq. (1.2) have the order zero? From the definition of the type of an entire function and the assumptions of Theorem B or Theorem D, we know that in Theorems B and D there exists at least one coefficient $a_{l}$ such that $\sigma\left(a_{l}\right)>0$.

Question 1.3 What can be said if there exists more than one coefficient having the order $\infty$ in Eq. (1.2)?

The main purpose of this paper is to investigate the above questions for Eq. (1.2). The remainder of the paper investigates the properties of meromorphic solutions of a nonhomogeneous linear difference equation corresponding to (1.2).

Theorem 1.1 Let $a_{j}(z)=A_{j}(z) e^{P_{j}(z)}(j=0,1, \ldots, k)$, where $P_{j}(z)=\alpha_{j n} z^{n}+\cdots+\alpha_{j 0}$ are polynomials with degree $n(\geq 1), A_{j}(z)(\not \equiv 0)$ are entire functions of $\sigma\left(A_{j}\right)<n$. If $\alpha_{j n}(j=0,1, \ldots, k)$ are distinct complex numbers, then every meromorphic solution $f(\not \equiv 0)$ of Eq. (1.2) satisfies $\sigma(f) \geq \max _{0 \leq j \leq k}\left\{\sigma\left(a_{j}\right)\right\}+1$.

Theorem 1.2 Let $a_{j}(z)=A_{j}(z) e^{P_{j}(z)}+D_{j}(z)(j=0,1, \ldots, k)$, where $A_{j}(z), P_{j}(z)$ satisfy the hypothesis of Theorem 1.1, $D_{j}(z)$ are entire functions with $\sigma\left(D_{j}\right)<n$. If $\alpha_{j n}(j=0,1, \ldots, k)$ are distinct complex numbers, then every meromorphic solution $f(\not \equiv 0)$ of Eq. (1.2) satisfies $\sigma(f) \geq \max _{0 \leq j \leq k}\left\{\sigma\left(a_{j}\right)\right\}+1$.

Remark 1.1 In Theorems 1.1 and 1.2, we have $\sigma\left(a_{0}\right)=\cdots=\sigma\left(a_{k}\right)$ and $\tau\left(a_{0}\right)=\cdots=\tau\left(a_{k}\right)$ if $\left|\alpha_{0 n}\right|=\cdots=\left|\alpha_{k n}\right|$. Therefore Theorems 1.1 and 1.2 are supplements of Theorem D.

Remark 1.2 From the proof of Theorems 1.1 and 1.2, we know that the same result also holds for Eq. (1.2) in the case when at least two coefficients have the form of $a_{j}(z)$ in Theorem 1.1 or 1.2 , and the orders of the others are less than $n$.

Theorem 1.3 Let $H$ be a complex set satisfying $\overline{\operatorname{dens}}\{r=|z|: z \in H\}>0$, and let $a_{0}(z), \ldots, a_{k}(z)$ be entire functions satisfying $\max _{0 \leq j \leq k}\left\{\sigma\left(a_{j}\right)\right\} \leq \sigma$. If there exists an integer $l(0 \leq l \leq k)$ such that for some constants $0 \leq \alpha<\beta$ and $\delta>0$ sufficiently small,

$$
\begin{aligned}
& \left|a_{l}(z)\right| \geq \exp \left\{\beta r^{\sigma-\delta}\right\}, \\
& \left|a_{j}(z)\right| \leq \exp \left\{\alpha r^{\sigma-\delta}\right\}, \quad j=0, \ldots, k, j \neq l,
\end{aligned}
$$

as $z \rightarrow \infty$ for $z \in H$, then every meromorphic solution $f(\not \equiv 0)$ of Eq. (1.2) satisfies $\sigma(f) \geq$ $\sigma\left(a_{l}\right)+1$.

Remark 1.3 Note that $\sigma$ may be zero in Theorem 1.3.

Example 1.1 shows the sharpness of Theorems 1.1 and 1.3, where $H=\{z: \arg z=0\}$. The following example illustrates the sharpness of Theorem 1.2. 
Example 1.2 The difference equation

$$
\left(z^{2} e^{-2 z-3}+e^{-4}\right) f(z+1)-\left(e^{2 z-1}+z^{2}\right) f(z)=0
$$

admits a solution $f(z)=e^{(z+1)^{2}}$, where $a_{1}(z)=z^{2} e^{-2 z-3}+e^{-4}, a_{0}(z)=-\left(e^{2 z-1}+z^{2}\right)$ satisfy the hypothesis of Theorem 1.2 and $\sigma\left(a_{1}\right)=\sigma\left(a_{0}\right), \tau\left(a_{1}\right)=\tau\left(a_{0}\right)$. Here $\sigma(f)=2=$ $\max \left\{\sigma\left(a_{0}\right), \sigma\left(a_{1}\right)\right\}+1$.

When there exists more than one coefficient having the order $\infty$ in Eq. (1.2), we obtain the following result. Note that in this case Theorem D is invalid.

Theorem 1.4 Let $a_{0}, a_{1}, \ldots, a_{k}$ be entire functions. If there exists an integer $l(0 \leq l \leq k)$ such that

$$
\begin{aligned}
& \max \left\{\sigma_{2}\left(a_{j}\right): j=0, \ldots, k, j \neq l\right\} \leq \sigma_{2}\left(a_{l}\right) \quad\left(0<\sigma_{2}\left(a_{l}\right)<\infty\right), \\
& \max \left\{\tau_{2}\left(a_{j}\right): \sigma_{2}\left(a_{j}\right)=\sigma_{2}\left(a_{l}\right)\right\}<\tau_{2}\left(a_{l}\right) \quad\left(0<\tau_{2}\left(a_{l}\right)<\infty\right),
\end{aligned}
$$

then every meromorphic solution $f(\not \equiv 0)$ of Eq. (1.2) satisfies $\sigma(f)=\infty$ and $\sigma_{2}(f) \geq \sigma_{2}\left(a_{l}\right)$.

Next we consider the properties of meromorphic solutions of the non-homogeneous linear difference equation corresponding to (1.2)

$$
a_{k}(z) f(z+k)+\cdots+a_{1}(z) f(z+1)+a_{0}(z) f(z)=F(z)
$$

where $F(z)(\not \equiv 0)$ is an entire function.

Theorem 1.5 Let $a_{j}(z)(j=0, \ldots, k)$ satisfy the hypothesis of Theorem 1.1 or Theorem 1.2, and let $F(z)$ be an entire function of $\sigma(F)<n$. Then at most one meromorphic solution $f_{0}$ of Eq. (1.5) satisfies $\max _{0 \leq j \leq k}\left\{\sigma\left(a_{j}\right)\right\} \leq \sigma\left(f_{0}\right)<\max _{0 \leq j \leq k}\left\{\sigma\left(a_{j}\right)\right\}+1$ and $\max \left\{\lambda\left(f_{0}\right), \lambda\left(1 / f_{0}\right)\right\}=$ $\sigma\left(f_{0}\right)$, the other solutions $f$ satisfy $\sigma\left(f_{0}\right) \geq \max _{0 \leq j \leq k}\left\{\sigma\left(a_{j}\right)\right\}+1$.

Theorem 1.6 Let $a_{j}(z)(j=0, \ldots, k)$ satisfy the hypothesis of Theorem 1.4 , and let $F(z)$ be an entire function. Then

(i) If $\sigma_{2}(F)<\sigma_{2}\left(a_{l}\right)$ or $\sigma_{2}(F)=\sigma_{2}\left(a_{l}\right), \tau_{2}(F)<\tau_{2}\left(a_{l}\right)$, then every meromorphic solution $f$ ( $\not \equiv 0)$ of Eq. (1.5) satisfies $\sigma(f)=\infty$ and $\sigma_{2}(f) \geq \sigma_{2}\left(a_{l}\right)$.

(ii) If $\sigma_{2}(F)>\sigma_{2}\left(a_{l}\right)$, then every meromorphic solution $f$ ( $\left.\not \equiv 0\right)$ of Eq. (1.5) satisfies $\sigma(f)=\infty$ and $\sigma_{2}(f) \geq \sigma_{2}(F)$.

\section{Lemmas}

Lemma 2.1 [8] Let $\eta_{1}, \eta_{2}$ be two arbitrary complex numbers, and let $f(z)$ be a meromorphic function offinite order $\sigma$. Let $\varepsilon>0$ be given, then there exists a subset $E \subset(0,+\infty)$ with finite logarithmic measure such that for all $|z|=r \notin E \cup[0,1]$, we have

$$
\exp \left\{-r^{\sigma-1+\varepsilon}\right\} \leq\left|\frac{f\left(z+\eta_{1}\right)}{f\left(z+\eta_{2}\right)}\right| \leq \exp \left\{r^{\sigma-1+\varepsilon}\right\}
$$


Lemma $2.2[20]$ Suppose that $P(z)=(\alpha+i \beta) z^{n}+\cdots(\alpha, \beta$ are real numbers, $|\alpha|+|\beta| \neq 0)$ is a polynomial with degree $n \geq 1, A(z)(\not \equiv 0)$ is an entire function with $\sigma(A)<n$. Set $g(z)=$ $A(z) e^{P(z)}, z=r e^{i \theta}, \delta(P, \theta)=\alpha \cos n \theta-\beta \sin n \theta$. Then for any given $\varepsilon>0$, there exists a set $E_{1} \subset[0,2 \pi)$ that has linear measure zero, such that for any $\theta \in[0,2 \pi) \backslash\left(E_{1} \cup E_{2}\right)$, there is $R>0$ such that for $|z|=r>R$, we have

(i) if $\delta(P, \theta)>0$, then

$$
\exp \left\{(1-\varepsilon) \delta(P, \theta) r^{n}\right\}<\left|g\left(r e^{i \theta}\right)\right|<\exp \left\{(1+\varepsilon) \delta(P, \theta) r^{n}\right\}
$$

(ii) if $\delta(P, \theta)<0$, then

$$
\exp \left\{(1+\varepsilon) \delta(P, \theta) r^{n}\right\}<\left|g\left(r e^{i \theta}\right)\right|<\exp \left\{(1-\varepsilon) \delta(P, \theta) r^{n}\right\}
$$

where $E_{2}=\{\theta \in[0,2 \pi): \delta(P, \theta)=0\}$ is a finite set.

Lemma 2.3 [21] Let $f(z)$ be an entire function of order $\sigma(f)=\sigma<+\infty$. Then for any given $\varepsilon>0$, there is a set $E \subset[1,+\infty)$ having finite linear measure such that for all $z$ satisfying $|z|=r \notin[0,1] \cup E$ and $r$ sufficiently large, we have

$$
\exp \left\{-r^{\sigma+\varepsilon}\right\} \leq|f(z)| \leq \exp \left\{r^{\sigma+\varepsilon}\right\}
$$

Lemma 2.4 [15] Let $f$ be a non-constant meromorphic function, $c \in \mathbb{C}, \delta<1$ and $\varepsilon>0$. Then

$$
m\left(r, \frac{f(z+c)}{f(z)}\right)=o\left(\frac{T(r+|c|, f)^{1+\varepsilon}}{r^{\delta}}\right)
$$

for all r outside of a possible exceptional set E with finite logarithmic measure $\int_{E} \frac{d r}{r}<\infty$.

Remark 2.1 By [5], we know that

$$
\begin{aligned}
(1+o(1)) T(r-|h|, f(z)) & \leq T(r, f(z+h)) \\
& \leq(1+o(1)) T(r+|h|, f(z)) \quad\left(r>r_{0}>0\right)
\end{aligned}
$$

where $h \in \mathbb{C}$. So, by (2.1) and (2.2), we immediately have

$$
m\left(r, \frac{f(z+c)}{f(z+h)}\right)=o\left(\frac{T(r+|c-h|+|h|, f)^{1+\varepsilon}}{r^{\delta}}\right)
$$

for all $r$ outside of a possible exceptional set $E$ with finite logarithmic measure $\int_{E} \frac{d r}{r}<\infty$.

Lemma 2.5 [4] Let $f$ be a meromorphic function with hyper-order $0<\sigma_{2}(f)<\infty$ and hyper-type $0<\tau_{2}(f)<\infty$, then for any given $\beta<\tau_{2}(f)$, there exists a subset $E \subset[1,+\infty)$ of infinite logarithmic measure such that $T(r, f)>\beta r^{\sigma_{2}(f)}$ holds for all $r \in E$.

Lemma 2.6 Let $G(z)=\sum_{j=1}^{k} B_{j}(z) e^{P_{j}(z)}$, where $P_{j}(z)=\alpha_{j n} z^{n}+\cdots+\alpha_{j 0}$ are polynomials with degree $n(\geq 1), B_{j}(z)(\not \equiv 0)$ are meromorphic functions of $\sigma\left(B_{j}\right)<n$. If $\alpha_{j n}(j=1, \ldots, k)$ are distinct complex numbers, then $\sigma(G)=n$. 
Proof By the Weierstrass factorization, we obtain

$$
B_{j}(z)=\frac{B_{j 1}(z)}{B_{j 2}(z)},
$$

where $B_{j 1}(z), B_{j 2}(z)$ are entire functions of order less than $n$. Let $Q(z)=\prod_{j=1}^{k} B_{j 2}(z), H(z)=$ $Q(z) G(z)$, then $\sigma(Q)<n$ and by $(2.4)$, we get

$$
H(z)=\sum_{j=1}^{k} D_{j}(z) e^{\alpha_{j n} z^{n}},
$$

where $D_{j}(z)=Q(z) B_{j}(z) e^{P_{j}(z)-\alpha_{j n} z^{n}}$ are entire functions of $\sigma\left(D_{j}\right)<n$.

Next we prove that $\sigma(H)=n$. Set $\sigma\left(D_{j}\right)=\sigma_{j}, \arg \alpha_{j n}=\varphi_{j}(1 \leq j \leq k)$. By Lemma 2.3, for any given $\varepsilon\left(0<\varepsilon<n-\max _{1 \leq j \leq k} \sigma_{j}\right)$, there is a set $E \subset[1,+\infty)$ having finite linear measure such that for all $z$ satisfying $|z|=r \notin[0,1] \cup E$ and $r$ sufficiently large, we have

$$
\exp \left\{-r^{\sigma_{j}+\varepsilon}\right\} \leq\left|D_{j}(z)\right| \leq \exp \left\{r^{\sigma_{j}+\varepsilon}\right\} \quad(1 \leq j \leq k)
$$

Without loss of generality, suppose that $\left|\alpha_{l n}\right| \geq \max \left\{\left|\alpha_{j n}\right|: 1 \leq j \leq k, j \neq l\right\}$. Let $\theta_{0}$ satisfy $\cos \left(\varphi_{l}+n \theta_{0}\right)=1$. Then by (2.5) and (2.6), for $z=r e^{i \theta_{0}}$ satisfying $|z|=r \notin[0,1] \cup E$ and $r$ sufficiently large, we have

$$
\begin{aligned}
|H(z)| \geq\left|D_{l}(z)\right|\left|e^{\alpha_{l n} z^{n}}\right|-\sum_{\substack{j=1 \\
j \neq l}}^{k}\left|D_{j}(z)\right|\left|e^{\alpha_{j n} z^{n}}\right| \\
\geq \exp \left\{-r^{\sigma_{l}+\varepsilon}\right\} \exp \left\{\left|\alpha_{l n}\right| r^{n}\right\}-\sum_{\substack{j=1 \\
j \neq l}}^{k} \exp \left\{r^{\sigma_{j}+\varepsilon}\right\} \exp \left\{\left|\alpha_{j n}\right| r^{n} \cos \left(\varphi_{j}+n \theta_{0}\right)\right\} \\
=\exp \left\{\left|\alpha_{l n}\right| r^{n}-r^{\sigma_{l}+\varepsilon}\right\}\left[1-\sum_{\substack{j=1 \\
j \neq l}}^{k} \exp \left\{\left(\left|\alpha_{j n}\right| \cos \left(\varphi_{j}+n \theta_{0}\right)-\left|\alpha_{l n}\right|\right) r^{n}\right\}\right. \\
\\
\left.\quad \cdot \exp \left\{r^{\sigma_{j}+\varepsilon}+r^{\sigma_{l}+\varepsilon}\right\}\right] .
\end{aligned}
$$

We discuss the following two cases.

Case 1. $\left|\alpha_{l n}\right|>\left|\alpha_{j n}\right|$ for $1 \leq j \leq k, j \neq l$. Then by (2.7), for $z=r e^{i \theta_{0}}$ satisfying $|z|=r \notin$ $[0,1] \cup E$ and $r$ sufficiently large, we have

$$
|H(z)| \geq \exp \left\{\left|\alpha_{\ln }\right| r^{n}-r^{\sigma_{l}+\varepsilon}\right\}(1-o(1))
$$

Case 2. Among $\alpha_{j n}(1 \leq j \leq k)$ there exist $\alpha_{i_{1} n}, \ldots, \alpha_{i_{m} n}\left(i_{j} \in\{1, \ldots, k\} \backslash\{l\}\right)$ such that $\left|\alpha_{i_{1} n}\right|=\cdots=\left|\alpha_{i_{m} n}\right|=\left|\alpha_{l n}\right|$. Since $\alpha_{j n}(1 \leq j \leq k)$ are distinct non-zero complex numbers, we have

$$
\cos \left(\varphi_{i_{1}}+\theta_{0}\right)<1, \quad \ldots, \quad \cos \left(\varphi_{i_{m}}+\theta_{0}\right)<1 .
$$

Hence by (2.7), we also obtain (2.8). 
By (2.8), we get $\sigma(H) \geq n$. On the other hand, by the elementary order considerations, we have $\sigma(H) \leq n$. So, $\sigma(H)=n$. Then by $G(z)=H(z) / Q(z)$ and $\sigma(Q)<n$, we get $\sigma(G)=n$.

\section{Proofs of the results}

Proof of Theorem 1.1 Let $f(\not \equiv 0)$ be a meromorphic solution of (1.2). Suppose that $\sigma(f)<$ $n+1$, then by Lemma 2.1 , for any given $\varepsilon>0$, there exists a set $E \subset(0,+\infty)$ with finite logarithmic measure such that for all $|z|=r \notin E \cup[0,1]$, we have

$$
\left|\frac{f(z+j)}{f(z+l)}\right| \leq \exp \left\{r^{\sigma(f)-1+\varepsilon}\right\} \quad(j=0, \ldots, k, j \neq l) .
$$

Set $z=r e^{i \theta}, \alpha_{j n}=\left|\alpha_{j n}\right| e^{i \varphi_{j}}$ and $\delta\left(P_{j}, \theta\right)=\left|\alpha_{j n}\right| \cos \left(\varphi_{j}+n \theta\right)(j=0, \ldots, k)$. Then $E_{1}=\{\theta \in$ $\left.[0,2 \pi): \delta\left(P_{j}, \theta\right)=0, j=0, \ldots, k\right\} \cup\left\{\theta \in[0,2 \pi): \delta\left(P_{j}-P_{i}, \theta\right)=0,0 \leq i<j \leq k\right\}$ is a set of linear measure zero. Considering each $a_{j}(z)=A_{j}(z) e^{P_{j}(z)}$, by Lemma 2.2, for the above $\varepsilon>0$, there exists a set $F_{j} \subset[0,2 \pi)$ of linear measure zero such that for any $z=r e^{i \theta}$ satisfying $\theta \in[0,2 \pi) \backslash\left(E_{1} \cup F_{j}\right)$ and $r$ sufficiently large, we have

(i) if $\delta\left(P_{j}, \theta\right)>0$, then

$$
\exp \left\{(1-\varepsilon) \delta\left(P_{j}, \theta\right) r^{n}\right\}<\left|a_{j}\left(r e^{i \theta}\right)\right|<\exp \left\{(1+\varepsilon) \delta\left(P_{j}, \theta\right) r^{n}\right\}
$$

(ii) if $\delta\left(P_{j}, \theta\right)<0$, then

$$
\exp \left\{(1+\varepsilon) \delta\left(P_{j}, \theta\right) r^{n}\right\}<\left|a_{j}\left(r e^{i \theta}\right)\right|<\exp \left\{(1-\varepsilon) \delta\left(P_{j}, \theta\right) r^{n}\right\} .
$$

Set $E_{2}=\bigcup_{j=0}^{k} F_{j}$, then $E_{2}$ is a set of linear measure zero. Since $\alpha_{j n}$ are distinct complex numbers, there exists only one $l \in\{0, \ldots, k\}$ such that $\delta\left(P_{l}, \theta\right)=\max \left\{\delta\left(P_{j}, \theta\right): j=0, \ldots, k\right\}$ for any $\theta \in[0,2 \pi) \backslash\left(E_{1} \cup E_{2}\right)$. Now we take a ray $\arg z=\theta_{0} \in[0,2 \pi) \backslash\left(E_{1} \cup E_{2}\right)$ such that $\delta\left(P_{l}, \theta_{0}\right)>0$. Let $\delta_{1}=\delta\left(P_{l}, \theta_{0}\right), \delta_{2}=\max \left\{\delta\left(P_{j}, \theta_{0}\right): j=0, \ldots, k, j \neq l\right\}$, then $\delta_{1}>\delta_{2}$. We discuss the following two cases.

Case $1 . \delta_{2}>0$. We rewrite (1.1) in the form

$$
-a_{l}(z)=\sum_{\substack{j=0 \\ j \neq l}}^{k} a_{j}(z) \frac{f(z+j)}{f(z+l)} .
$$

By (3.1), (3.2) and (3.4), we get for $z=r e^{i \theta_{0}}$ and sufficiently large $r \notin E \cup[0,1]$,

$$
\begin{aligned}
\exp \left\{(1-\varepsilon) \delta_{1} r^{n}\right\} & \leq\left|a_{l}\left(r e^{i \theta_{0}}\right)\right| \leq \sum_{\substack{j=0 \\
j \neq l}}^{k} \exp \left\{(1+\varepsilon) \delta\left(P_{j}, \theta_{0}\right) r^{n}\right\} \exp \left\{r^{\sigma(f)-1+\varepsilon}\right\} \\
& \leq k \exp \left\{(1+\varepsilon) \delta_{2} r^{n}\right\} \exp \left\{r^{\sigma(f)-1+\varepsilon}\right\} .
\end{aligned}
$$

When $0<2 \varepsilon<\min \left\{\frac{\delta_{1}-\delta_{2}}{\delta_{1}+\delta_{2}}, n+1-\sigma(f)\right\}$, by (3.5), we get

$$
\exp \left\{\frac{\delta_{1}-\delta_{2}}{2} r^{n}\right\} \leq k \exp \left\{r^{\sigma(f)-1+\varepsilon}\right\} \text {. }
$$

This is impossible. 
Case 2. $\delta_{2}<0$. By (3.1), (3.3) and (3.4), we get for $z=r e^{i \theta_{0}}$ and sufficiently large $r \notin$ $E \cup[0,1]$,

$$
\begin{aligned}
\exp \left\{(1-\varepsilon) \delta_{1} r^{n}\right\} & \leq\left|a_{l}\left(r e^{i \theta_{0}}\right)\right| \leq \sum_{\substack{j=0 \\
j \neq l}}^{k} \exp \left\{(1-\varepsilon) \delta\left(P_{j}, \theta_{0}\right) r^{n}\right\} \exp \left\{r^{\sigma(f)-1+\varepsilon}\right\} \\
& \leq k \exp \left\{r^{\sigma(f)-1+\varepsilon}\right\} .
\end{aligned}
$$

This is a contradiction. Hence we get $\sigma(f) \geq n+1=\max _{0 \leq j \leq k}\left\{\sigma\left(a_{j}\right)\right\}+1$.

Proof of Theorem 1.2 By Lemmas 2.2 and 2.3, we know that for any given $\varepsilon>0$, there is a set $E \subset[1,+\infty)$ having finite linear measure such that for all $z$ satisfying $|z|=r \notin[0,1] \cup E$ and $r$ sufficiently large, we have

$$
\exp \left\{(1-\varepsilon) \delta\left(P_{j}, \theta\right) r^{n}\right\}<\left|a_{j}\left(r e^{i \theta}\right)\right|<\exp \left\{(1+\varepsilon) \delta\left(P_{j}, \theta\right) r^{n}\right\}
$$

if $\delta\left(P_{j}, \theta\right)>0$, and

$$
\left|a_{j}\left(r e^{i \theta}\right)\right|<\exp \left\{r^{\sigma\left(D_{j}\right)+\varepsilon}\right\}
$$

if $\delta\left(P_{j}, \theta\right)<0$. Then using the similar argument to that of Theorem 1.1 and only replacing (3.2) (or (3.3)) by (3.6) (or (3.7)), we can prove Theorem 1.2.

Proof of Theorem 1.3 Let $f(\not \equiv 0)$ be a meromorphic solution of (1.2). Suppose that $\sigma(f)<$ $\sigma+1$, then by Lemma 2.1, for any given $\varepsilon(0<\varepsilon<\sigma+1-\sigma(f)-2 \delta)$, there exists a set $E \subset(0,+\infty)$ with finite logarithmic measure such that for all $|z|=r \notin E \cup[0,1]$, we have

$$
\left|\frac{f(z+j)}{f(z+l)}\right| \leq \exp \left\{r^{\sigma(f)-1+\varepsilon}\right\}<\exp \left\{r^{\sigma-2 \delta}\right\} \quad(j=0, \ldots, k, j \neq l) .
$$

Rewrite (1.1) in the form

$$
-1=\sum_{\substack{j=0 \\ j \neq l}}^{k} \frac{a_{j}(z) f(z+j)}{a_{l}(z) f(z+l)} .
$$

Since $E \cup[0,1]$ has finite logarithmic measure, the density of $E \cup[0,1]$ is zero. Hence (1.3) and (1.4) also hold for $z \in H \backslash E \cup[0,1]$. Substituting (1.3), (1.4) and (3.8) into (3.9), we get for $z \in H \backslash E \cup[0,1]$,

$$
\begin{aligned}
1 & \leq \sum_{\substack{j=0 \\
j \neq l}}^{k} \exp \left\{(\alpha-\beta) r^{\sigma-\delta}\right\} \exp \left\{r^{\sigma-2 \delta}\right\} \\
& \rightarrow 0
\end{aligned}
$$

a contradiction. Hence we get $\sigma(f) \geq \sigma+1$. By the assumptions of Theorem 1.3, we know that $\sigma\left(a_{l}\right)=\sigma$. So, $\sigma(f) \geq \sigma\left(a_{l}\right)+1$. 
Proof of Theorem 1.4 Let $f(\not \equiv 0)$ be a meromorphic solution of (1.2). By (1.2) we get

$$
-a_{l}(z)=\sum_{\substack{j=0 \\ j \neq l}}^{k} a_{j}(z) \frac{f(z+j)}{f(z+l)}
$$

By Lemma 2.4 and (3.10), we get

$$
\begin{aligned}
m\left(r, a_{l}(z)\right) & \leq \sum_{\substack{j=0 \\
j \neq l}}^{k} m\left(r, a_{j}(z)\right)+\sum_{\substack{j=0 \\
j \neq l}}^{k} m\left(r, \frac{f(z+j)}{f(z+l)}\right)+O(1) \\
& =\sum_{\substack{j=0 \\
j \neq l}}^{k} m\left(r, a_{j}(z)\right)+\sum_{\substack{j=0 \\
j \neq l}}^{k} o\left(\frac{T(r+|l|+|j-l|, f)^{1+\varepsilon}}{r^{\delta}}\right)+O(1) \\
& =\sum_{\substack{j=0 \\
j \neq l}}^{k} T\left(r, a_{j}(z)\right)+o\left(\frac{T(r+2 k, f)^{1+\varepsilon}}{r^{\delta}}\right)+O(1)
\end{aligned}
$$

for $r \rightarrow \infty, r \notin E$, where $E$ is a set of finite logarithmic measure.

Let $\beta_{1}, \beta_{2}$ be two real numbers such that $\max \left\{\tau_{2}\left(a_{j}\right): \sigma_{2}\left(a_{j}\right)=\sigma_{2}\left(a_{l}\right)\right\}<\beta_{1}<\beta_{2}<\tau_{2}\left(a_{l}\right)$. Then by Lemma 2.5 , we know that there exists a set $H$ of infinite logarithmic measure such that

$$
T\left(r, a_{l}\right)>\exp \left\{\beta_{2} r^{\sigma_{2}\left(a_{l}\right)}\right\}
$$

holds for all $r \in H$. Therefore we can take a sequence $\left\{r_{n}\right\}$ such that $r_{n} \in H \backslash E, r_{n} \rightarrow \infty$ and

$$
T\left(r_{n}, a_{l}\right)>\exp \left\{\beta_{2} r_{n}^{\sigma_{2}\left(a_{l}\right)}\right\}
$$

holds for sufficiently large $r_{n}$.

On the other hand, if $\sigma_{2}\left(a_{j}\right)<\sigma_{2}\left(a_{l}\right)$, then for any given $\varepsilon>0$ and sufficiently large $r_{n}$, we have

$$
T\left(r_{n}, a_{j}\right)<\exp \left\{r_{n}^{\sigma_{2}\left(a_{j}\right)+\varepsilon}\right\}<\exp \left\{\beta_{1} r_{n}^{\sigma_{2}\left(a_{l}\right)}\right\}
$$

if $\max \left\{\tau_{2}\left(a_{j}\right): \sigma_{2}\left(a_{j}\right)=\sigma_{2}\left(a_{l}\right)\right\}<\tau_{2}\left(a_{l}\right)$, then for sufficiently large $r_{n}$, we have

$$
T\left(r_{n}, a_{j}\right)<\exp \left\{\beta_{1} r_{n}^{\sigma_{2}\left(a_{l}\right)}\right\}
$$

Then substituting (3.12), (3.13) (or (3.14)) into (3.11), we get

$$
\begin{aligned}
\exp \left\{\beta_{2} r_{n}^{\sigma_{2}\left(a_{l}\right)}\right\} & <T\left(r_{n}, a_{l}\right)=m\left(r_{n}, a_{l}\right) \\
& <k \exp \left\{\beta_{1} r_{n}^{\sigma_{2}\left(a_{l}\right)}\right\}+o\left(\frac{T\left(r_{n}+2 k, f\right)^{1+\varepsilon}}{r_{n}^{\delta}}\right) .
\end{aligned}
$$


Hence by (3.15), for sufficiently large $r_{n}$, we have

$$
(1-o(1)) \exp \left\{\beta_{2} r_{n}^{\sigma_{2}\left(a_{l}\right)}\right\}=o\left(\frac{T\left(r_{n}+2 k, f\right)^{1+\varepsilon}}{r_{n}^{\delta}}\right)
$$

Then by (3.16), we get $\sigma(f)=\infty$ and $\sigma_{2}(f) \geq \sigma_{2}\left(a_{l}\right)$.

Proof of Theorem 1.5 Let $f(\not \equiv 0)$ be a meromorphic solution of (1.5). Suppose that $\sigma(f)<\max _{0 \leq j \leq k}\left\{\sigma\left(a_{j}\right)\right\}$, then by Lemma 2.6 we obtain $\sigma(F)=\sigma\left(\sum_{j=0}^{k} a_{j}(z) f(z+j)\right)=n$. This contradicts $\sigma(F)<n$. Therefore we have $\sigma(f) \geq \max _{0 \leq j \leq k}\left\{\sigma\left(a_{j}\right)\right\}$.

Suppose that there exist two distinct meromorphic solutions $f_{1}(\not \equiv 0), f_{2}(\not \equiv 0)$ of Eq. (1.5) such that $\max \left\{\sigma\left(f_{1}\right), \sigma\left(f_{2}\right)\right\}<\max _{0 \leq j \leq k}\left\{\sigma\left(a_{j}\right)\right\}+1$. Then $f_{1}-f_{2}$ is a meromorphic solution of the homogeneous linear difference equation corresponding to (1.5), and $\sigma\left(f_{1}-f_{2}\right)<\max _{0 \leq j \leq k}\left\{\sigma\left(a_{j}\right)\right\}+1$. By Theorem 1.1 or Theorem 1.2, we get a contradiction. So, Eq. (1.5) has at most one meromorphic solution $f_{0}$ satisfying $\max _{0 \leq j \leq k}\left\{\sigma\left(a_{j}\right)\right\} \leq \sigma\left(f_{0}\right)<$ $\max _{0 \leq j \leq k}\left\{\sigma\left(a_{j}\right)\right\}+1$.

Next we prove $\max \left\{\lambda\left(f_{0}\right), \lambda\left(1 / f_{0}\right)\right\}=\sigma\left(f_{0}\right)$ in the case $\sigma\left(f_{0}\right)=\max _{0 \leq j \leq k}\left\{\sigma\left(a_{j}\right)\right\}$. Suppose that $\max \left\{\lambda\left(f_{0}\right), \lambda\left(1 / f_{0}\right)\right\}<\sigma\left(f_{0}\right)$, then by the Weierstrass factorization, we obtain

$$
f_{0}(z)=\frac{g_{1}(z)}{g_{2}(z)} e^{Q(z)}
$$

where $Q(z)=\beta z^{n}+\cdots$ is a polynomial of degree $n, g_{1}(z)$ and $g_{2}(z)$ are entire functions of $\sigma\left(g_{1}\right)=\lambda\left(g_{1}\right)=\lambda\left(f_{0}\right), \sigma\left(g_{2}\right)=\lambda\left(g_{2}\right)=\lambda\left(1 / f_{0}\right)$. Let $g(z)=\frac{g_{1}(z)}{g_{2}(z)} e^{Q(z)-\beta z^{n}}$ and $B_{j}(z)=$ $A_{j}(z) e^{P_{j}(z)-\alpha_{j n} z^{n}}$. Substituting (3.17) into (1.5), we get

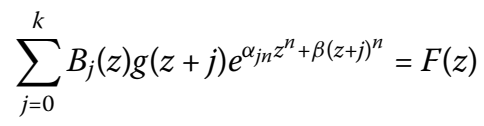

in the case $a_{j}=A_{j} e^{P_{j}}$. Since $\alpha_{j n}$ are distinct complex numbers, by Lemma 2.6, we obtain that the order of the left-hand side of (3.18) is $n$. This contradicts $\sigma(F)<n$. For $a_{j}=A_{j} e^{P_{j}}+D_{j}$, by using a similar to the above argument, we also obtain a contradiction.

It is obvious that $\max \left\{\lambda\left(f_{0}\right), \lambda\left(1 / f_{0}\right)\right\}=\sigma\left(f_{0}\right)$ provided that $\max _{0 \leq j \leq k}\left\{\sigma\left(a_{j}\right)\right\}<\sigma\left(f_{0}\right)<$ $\max _{0 \leq j \leq k}\left\{\sigma\left(a_{j}\right)\right\}+1$. Therefore we have $\max \left\{\lambda\left(f_{0}\right), \lambda\left(1 / f_{0}\right)\right\}=\sigma\left(f_{0}\right)$.

Proof of Theorem 1.6 First we consider the case $\sigma_{2}(F)<\sigma_{2}\left(a_{l}\right)$ or $\sigma_{2}(F)=\sigma_{2}\left(a_{l}\right), \tau_{2}(F)<$ $\tau_{2}\left(a_{l}\right)$. Let $f(\not \equiv 0)$ be a meromorphic solution of (1.5). By (1.5), (2.2) and (2.3), we get

$$
\begin{aligned}
m\left(r, a_{l}(z)\right) \leq & m\left(r, \frac{F(z)}{f(z+l)}\right)+\sum_{\substack{j=0 \\
j \neq l}}^{k} m\left(r, a_{j}(z)\right)+\sum_{\substack{j=0 \\
j \neq l}}^{k} m\left(r, \frac{f(z+j)}{f(z+l)}\right)+O(1) \\
\leq & T(r, F(z))+T(r, f(z+l))+\sum_{\substack{j=0 \\
j \neq l}}^{k} m\left(r, a_{j}(z)\right) \\
& +\sum_{\substack{j=0 \\
j \neq l}}^{k} o\left(\frac{T(r+|l|+|j-l|, f)^{1+\varepsilon}}{r^{\delta}}\right)+O(1)
\end{aligned}
$$




$$
\begin{aligned}
\leq & T(r, F(z))+(1+o(1)) T(r+|l|, f(z))+\sum_{\substack{j=0 \\
j \neq l}}^{k} T\left(r, a_{j}(z)\right) \\
& +o\left(\frac{T(r+2 k, f)^{1+\varepsilon}}{r^{\delta}}\right)+O(1)
\end{aligned}
$$

for $r \rightarrow \infty, r \notin E$, where $E$ is a set of finite logarithmic measure.

Let $\beta_{1}, \beta_{2}$ be two real numbers such that $\max \left\{\tau_{2}\left(a_{j}\right), \tau_{2}(F): \sigma_{2}\left(a_{j}\right)=\sigma_{2}\left(a_{l}\right)\right\}<\beta_{1}<\beta_{2}<$ $\tau_{2}\left(a_{l}\right)$. Then by Lemma 2.5 , we can take a sequence $\left\{r_{n}\right\}$ such that $r_{n} \in H \backslash E, r_{n} \rightarrow \infty$ and (3.12)-(3.14) also hold for sufficiently large $r_{n}$, where $H$ is defined by Lemma 2.5. On the other hand, for sufficiently large $r_{n}$ we have

$$
T\left(r_{n}, F\right)<\exp \left\{\beta_{1} r_{n}^{\sigma_{2}\left(a_{l}\right)}\right\}
$$

Substituting (3.12), (3.13) (or (3.14)) (3.20) into (3.19), we get

$$
\begin{aligned}
\exp \left\{\beta_{2} r_{n}^{\sigma_{2}\left(a_{l}\right)}\right\} & \leq T\left(r_{n}, a_{l}\right)=m\left(r_{n}, a_{l}\right) \\
& <(k+1) \exp \left\{\beta_{1} r_{n}^{\sigma_{2}\left(a_{l}\right)}\right\}+3 T\left(2 r_{n}, f\right)^{2} .
\end{aligned}
$$

Hence by (3.21), we get $\sigma(f)=\infty$ and $\sigma_{2}(f) \geq \sigma_{2}\left(a_{l}\right)$.

Next we consider the case $\sigma_{2}(F)>\sigma_{2}\left(a_{l}\right)$. Let $f(\not \equiv 0)$ be a meromorphic solution of (1.5). By (1.5) and (2.2), we get

$$
\begin{aligned}
T(r, F(z)) & \leq \sum_{j=0}^{k} T\left(r, a_{j}(z)\right)+\sum_{j=0}^{k} T(r, f(z+j))+O(1) \\
& \leq \sum_{j=0}^{k} T\left(r, a_{j}(z)\right)+2(k+1) T(r+k, f(z)) .
\end{aligned}
$$

By the definition of hyper-order, we know that there exists a sequence $\left\{r_{n}\right\}$ such that $r_{n} \rightarrow$ $\infty$, and for any given $\varepsilon\left(0<2 \varepsilon<\sigma_{2}(F)-\sigma_{2}\left(a_{l}\right)\right)$ and sufficiently large $r_{n}$, we have

$$
\begin{aligned}
& T\left(r_{n}, F\right)>\exp \left\{r_{n}^{\sigma_{2}(F)-\varepsilon}\right\}, \\
& T\left(r_{n}, a_{j}\right)<\exp \left\{r_{n}^{\sigma_{2}\left(a_{j}\right)+\varepsilon}\right\} \quad(j=0, \ldots, k) .
\end{aligned}
$$

Substituting (3.23), (3.24) into (3.22), we get $\sigma(f)=\infty$ and $\sigma_{2}(f) \geq \sigma_{2}(F)$.

\section{Competing interests}

The authors declare that they have no competing interests.

\section{Authors' contributions}

Both authors drafted the manuscript, read and approved the final manuscript.

\section{Acknowledgements}

This work is supported by the National Natural Science Foundation of China (No. 11201195, 11171119), the Natural Science Foundation of Jiangxi, China (No. 20122BAB201012, 20132BAB201008, 20122BAB211005), the STP of the Education Department of Jiangxi, China (No. GJJ12179). The authors thank the referee for his/her valuable suggestions to improve the present article. 


\section{References}

1. Hayman, WK: Meromorphic Functions. Clarendon Press, Oxford (1964)

2. Laine, I: Nevanlinna Theory and Complex Differential Equations. de Gruyter, Berlin (1993)

3. Yang, L: Value Distribution Theory and New Research. Science Press, Beijing (1982) (in Chinese)

4. Cao, TB, Xu, JF, Chen, ZX: On the meromorphic solutions of linear differential equations on the complex plane. J. Math. Anal. Appl. 364, 130-142 (2010)

5. Ablowitz, MJ, Halburd, R, Herbst, B: On the extension of the Painlevé property to difference equations. Nonlinearity 13, 889-905 (2000)

6. Bergweiler, W, Ishizaki, K, Yanagihara, N: Meromorphic solutions of some functional equations. Methods Appl. Anal. 5, 248-258 (1998)

7. Bergweiler, W, Ishizaki, K, Yanagihara, N: Growth of meromorphic solutions of some functional equations I. Aequ. Math. 63, 140-151 (2002)

8. Chiang, YM, Feng, SJ: On the Nevanlinna characteristic of $f(z+\eta)$ and difference equations in the complex plane. Ramanujan J. 16, 105-129 (2008)

9. Chiang, YM, Feng, SJ: On the growth of logarithmic differences, difference quotients and logarithmic derivatives of meromorphic functions. Trans. Am. Math. Soc. 361, 3767-3791 (2009)

10. Chen, ZX: Growth and zeros of meromorphic solution of some linear difference equations. J. Math. Anal. Appl. 373, 235-241 (2011)

11. Chen, ZX, Shon, $\mathrm{KH}$ : Value distribution of meromorphic solutions of certain difference Painlevé equations. J. Math Anal. Appl. 364, 556-566 (2010)

12. Chen, ZX: On growth, zeros and poles of meromorphic solutions of linear and nonlinear difference equations. Sci. China Ser. A 54, 2123-2133 (2011)

13. Chen, ZX, Shon, $\mathrm{KH}$ : On the growth and fixed points of solutions of second order differential equations with meromorphic coefficients. Acta Math. Sin. Engl. Ser. 21, 753-764 (2005)

14. Halburd, RG, Korhonen, R: Difference analogue of the lemma on the logarithmic derivative with applications to difference equations. J. Math. Anal. Appl. 314, 477-487 (2006)

15. Halburd, RG, Korhonen, R: Nevanlinna theory for the difference operator. Ann. Acad. Sci. Fenn., Math. 31, 463-478 (2006)

16. Halburd, RG, Korhonen, R: Meromorphic solutions of difference equations, integrability and the discrete Painlevé equations. J. Phys. A 40, 1-38 (2007)

17. Heittokangas, J, Korhonen, R, Laine, I, Rieppo, J, Tohge, K: Complex difference equations of Malmquist type. Comput. Methods Funct. Theory 1, 27-39 (2001)

18. Laine, I, Yang, CC: Clunie theorems for difference and q-difference polynomials. J. Lond. Math. Soc. 76, 556-566 (2007)

19. Wang, J: Growth and poles of meromorphic solutions of some difference equations. J. Math. Anal. Appl. 379, 367-377 (2011)

20. Halburd, RG, Korhonen, R: Finite-order meromorphic solutions and the discrete Painlevé equations. Proc. Lond. Math. Soc. 94, 443-474 (2007)

21. Chen, ZX: On the hyper order of solutions of some second order linear differential equation. Acta Math. Sin. Engl. Ser. 18, 79-88 (2002) (in Chinese)

doi:10.1186/1687-1847-2013-133

Cite this article as: Liu and Mao: On the meromorphic solutions of some linear difference equations. Advances in Difference Equations 2013 2013:133.

\section{Submit your manuscript to a SpringerOpen ${ }^{0}$ journal and benefit from:}

- Convenient online submission

- Rigorous peer review

- Immediate publication on acceptance

- Open access: articles freely available online

- High visibility within the field

- Retaining the copyright to your article 